This is the peer reviewed version of the following article: Macpherson, M. F., Davidson, R. S., Duncan, D. B., Lurz, P. W., Jarrott, A. and White, A. (2016), Incorporating habitat distribution in wildlife disease models: conservation implications for the threat of squirrelpox on the Isle of Arran. Animal Conservation, 19: 3-14, which has been published in final form at https://doi.org/10.1111/acv.12219. This article may be used for non-commercial purposes in accordance With Wiley Terms and Conditions for self-archiving. 


\title{
Incorporating habitat distribution in wildlife disease models: conservation implications for the threat of squirrelpox on the Isle of Arran.
}

\author{
Morag F. Macpherson ${ }^{\mathrm{a}, \mathrm{b}}$, Ross S. Davidson ${ }^{\mathrm{b}}$, Dugald B. Duncan ${ }^{\mathrm{a}}$, Peter W. Lurz ${ }^{\mathrm{c}}$, Andrew \\ Jarrott $^{\mathrm{d}}$ and Andy White ${ }^{\mathrm{a}}$

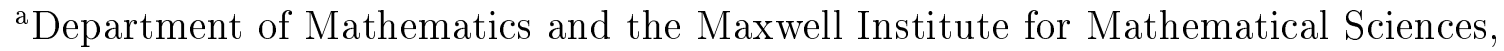 \\ Heriot-Watt University, Edinburgh EH14 4AS, UK \\ ${ }^{\mathrm{b}}$ Disease Systems, SRUC, West Mains Road, Edinburgh EH9 3JG, UK \\ ${ }^{\mathrm{c}}$ Royal (Dick) School of Veterinary Studies, The University of Edinburgh, Easter Bush \\ Campus, Midlothian EH25 9RG, UK \\ ${ }^{\mathrm{d}}$ Forestry Commission Scotland, Galloway Forest District, Creebridge, Newton Stewart, DG8 \\ $6 \mathrm{AJ}$
}

\begin{abstract}
Emerging infectious diseases are a substantial threat to native populations. The spread of disease through naive native populations will depend on both demographic and disease parameters, as well as on habitat suitability and connectivity. Using the potential spread of squirrelpox virus (SQPV) on the Isle of Arran as a case study, we develop mathematical models to examine the impact of an emerging disease on a population in a complex landscape of different habitat types. Furthermore, by considering a range of disease parameters, we infer more generally how complex landscapes interact with disease characteristics to determine the spread and persistence of disease. Specific findings indicate that a SQPV outbreak on Arran is likely to be short lived and localised to the point of introduction allowing recovery of red squirrels to pre-infection densities; this has important consequences for the conservation of red squirrels. More generally, we find the extent of disease spread is dependent on the rare passage of infection through poor quality corridors connecting good quality habitats. Acute, highly transmissible infectious diseases are predicted to spread rapidly causing high mortality. Nonetheless the disease typically fades out following local epidemics and is not supported in the long-term. A chronic infectious disease is predicted to spread more slowly but can remain endemic in the population. This allows the disease to spread more extensively in the long-term as it increases the chance of spread between poorly connected populations. Our results highlight how a detailed understanding of landscape connectivity is crucial when considering conservation strategies to protect native species from disease threats.
\end{abstract}




\section{Introduction}

The heterogeneous distribution and connectivity of habitat has a complex relationship with population abundance and persistence (Bienen, 2002). When habitat is sparse, isolated habitat patches can be formed dividing a population into several subpopulations (Hanski and Gilpin, 1991). Poor connectivity between patches may result in a reduction of movement between local populations which in turn can act to decrease patch biodiversity (Kareiva and Wennergren, 1995), fitness due to inbreeding (Chesser and Ryman, 1986) and population growth (Travis, 2003). Furthermore poor connectivity may lead to a higher probability of local extinction (Fahrig and Merriam, 1985; Henein and Merriam, 1990; Schumaker, 1996) and reduced likelihood of natural re-establishment via dispersal (Fahrig and Merriam, 1985; van Andel and Aronson, 2012). Evidence therefore supports the maintenance of dispersal corridors to promote growth and local population persistence and diversity (Beier and Noss, 1998; Dobson et al., 1999; Lookingbill et al., 2010; van Andel and Aronson, 2012). However, enhanced connectivity between subpopulations can increase the spread of infectious disease leading to detrimental impacts on native populations (Hess, 1994; Real and Biek, 2007).

Emerging infectious diseases (EIDs) contribute to species extinction (Smith et al., 2006) and are a global threat to biodiversity (Daszak, 2000; Daszak et al., 2001; Morse, 1995). Morse (1995) proposed that EIDs were caused by a two-step process: the deliberate or accidental introduction of non-native species and/or disease into a susceptible population, and their subsequent establishment within the new environment. The exposure of susceptible indigenous populations to novel and potentially fatal disease may cause population declines and in turn impact species competition, habitat and decrease biodiversity (Manchester and Bullock, 2000). One of the many examples of emergent infectious diseases (Daszak, 2000) is the fungal pathogen Chalara fraxinea, believed to have emerged in Poland in 1992 and has since caused widespread die back in Ash species (up to $90 \%$ mortality) across Europe (Worrell, 2012). Understanding the spread of EIDs may be crucial to the conservation of native species. Therefore, understanding how disease spreads in the environment can help reduce the impact of infectious disease in wildlife and humans (Patz et al., 2004).

The use of spatial frameworks in mathematical models to explore the impact of infectious disease in wildlife has been well established (Hanski and Gilpin, 1991; Hanski, 1998; Hess, 1996; Keeling, 1999; Real and Biek, 2007; Rushton et al., 2000). Previous theoretical studies which have examined infection dynamics have used classical Levins (Levins, 1969) metapopulation approaches (Hess, 1996; McCallum and Dobson, 2002), simulation methods with an idealised spatial set-up, such as patches connected in a linear or looped array (Hess, 1996; White et al., 2014), or a two-dimensional lattice of homogeneous patches (Cross et al., 2005). However, despite the heterogeneity of natural landscapes and habitats, the incorporation of detailed, realistic landscape structure has been less well explored (Ostfeld et al., 2005). A realistic representation of landscape structure may consist of a fragmented mixture of high and low quality subpopulations with different levels of inter-population 
connectivity. Moreover, for directly transmitted parasites, susceptible individuals must encounter an infected individual for infection to spread (Riley, 2007), thus the inclusion of geographical data in spatial disease models may identify corridors aiding the spread of infectious disease which in turn may be used to contain outbreaks (Beier and Noss, 1998; Dobson et al., 1999; Riley, 2007). We therefore extend the work of previous studies by considering a heterogeneous spatial set-up derived from real habitat information. A key question will be to understand how results translate between idealised and realistic landscapes.

To illustrate the importance of modelling disease spread in complex environments we examine the impact of habitat distribution and connectivity on the spread of infectious disease in the Eurasian red squirrel (Sciurus vulgaris) on the Isle of Arran (Figure 1(a)). The Eurasian red squirrel has been in decline in mainland UK since the introduction of the North American grey squirrels (Sciurus carolinensis) in the late 19th century. Initially competition alone was blamed for causing the reduction in the native population. However it is now accepted that disease-mediated invasion is a critical factor in the replacement of red squirrels (Daszak, 2000; Rushton et al., 2000; Tompkins et al., 2003). The emerging squirrelpox virus (SQPV) is likely to have been introduced to the UK with the grey squirrels (since it is largely avirulent in greys) (Bosch and Lurz, 2012). Squirrelpox causes high mortality in red squirrels and is critical in explaining the rapid replacement of reds (Tompkins et al., 2003). To prevent species extinction the UK Biodiversity Action Plan (Joint Nature Conservation Committee, 2012) has designated 35 red squirrel strongholds (18 in Scotland and 17 in England) and it is therefore important to understand the threat of SQPV in these strongholds. One such stronghold, Arran, was chosen for its island status and lack of grey squirrels thus far (Lurz, 2012). Arran is roughly $32 \mathrm{~km}$ by $20 \mathrm{~km}$ in dimensions with 6803ha of fragmented woodland, predominately coniferous, with a mixture of high and low connectivity between local patches (Figure 1) (Lurz, 2012). Constructing a spatial disease framework based on red squirrels on Arran provides an ideal case study to examine the impact habitat connectivity has on the spread of infectious disease.

Our aims are therefore two-fold. Firstly, our case study provides species and location specific results that can directly inform conservation management and policy decisions. Secondly, we aim to provide a general assessment of the importance of replicating geographical information accurately in spatial models when analysing disease spread in complex landscapes. Therefore in addition to considering the spread of SQPV we modify the model to include a range of disease parameters - chronic and acute disease with high and low transmissibility - and so can infer how the complexity of the landscape interacts with disease characteristics to determine the spread and persistence of disease. Moreover, we include two levels of connectivity, one derived directly from geographical information software (GIS) data and one which is additionally supplemented with ground-truthing from local experts that indicate additional barriers to dispersal. We therefore provide a general understanding of how landscape connectivity and infectious disease properties influences disease spread and persistence. 


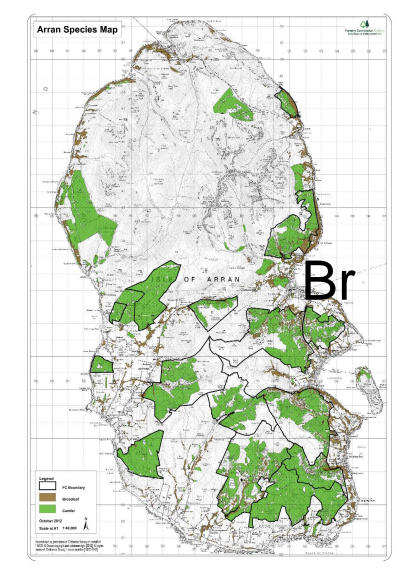

(a)

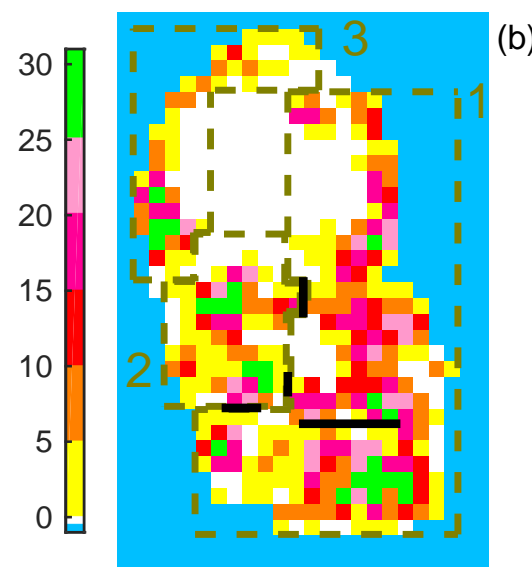

Figure 1: The Isle of Arran is shown in (a) (with permission from FCS) with the coniferous (green) and broadleaf (brown) woodland highlighted and ' $\mathrm{Br}$ ' represents the main port Brodick. The carrying capacity of red squirrels (per $\mathrm{km}^{2}$ ) is shown in (b) which uses upper density estimates of abundance of $0.36 \mathrm{ha}^{-1}$ in coniferous and $0.6 \mathrm{ha}^{-1}$ in broadleaf. Cells which are well connected are grouped together by the dashed olive blocks (labelled regions $1-3$ ); there is poor connectivity between the blocks. In addition, ground-truthing in collaboration with Forestry Commission Scotland, has identified routes which do not permit movement, identified by thick black lines.

\section{Methods}

\subsection{Red squirrel carrying capacity}

We extrapolated woodland data from the forest inventory maps for 2011 (provided by the Forestry Commission Scotland (FCS) website) using GRASS Geographical Information System (GIS) (Westervelt et al., 1992) (using similar methods to Rushton et al. (2000) and Lurz et al. (2003)). The island was divided into a $1 \mathrm{~km} \times 1 \mathrm{~km}$ grid - since $1 \mathrm{~km}$ is typically the maximum dispersal distance for red squirrels (Bosch and Lurz, 2012) and larger than the typical $150 \mathrm{~m}$ radius of core range activity (which is based on estimates of red squirrels home range of 9ha (Tonkin, 1983; Lurz, 1995; Bosch and Lurz, 2012)). Within each grid cell habitat data identifying no coverage (thus unable to support red squirrels), coniferous and broadleaf woodland was determined from inventory records (shown in Figure 1(a)). Published densities of red squirrel abundance in different tree species from (Lurz, 2012) was used to calculate the carrying capacity for each cell dependent on the quantity and type of woodland cover (Figure 1(b)). We used upper density estimates for abundance (which represents repeated high seed years and therefore a worst case scenario for disease spread) with results for lower density estimates (repeated poor seed years) shown in the Supplementary Information. 


\subsection{The Model}

We assume that any grey squirrels/disease introduced to Arran would be the result of an accidental or deliberate release at the main port (Brodick) on the island (Figure 1(a)). Contingency plans have been developed on Arran to trap and remove greys should they be sighted but this may not prevent disease transmission to the established local red squirrel population and so our initial conditions assume a local population of reds is infected. We base our approach on that of Tompkins et al. (2003), who modelled squirrelpox in both red and grey squirrels on mainland UK. Here we use the equivalent model for a population of red squirrels only, giving

$$
\begin{aligned}
& \frac{d S}{d t}=(a-q H) H-b S-\beta S I \\
& \frac{d I}{d t}=\beta S I-(b+\sigma) I
\end{aligned}
$$

for susceptible $(S)$ and infected $(I)$ individuals, in which the total population $H=S+I$. Reds are born susceptible with maximum reproduction rate $a$ and natural mortality rate $b$. We assume the carrying capacity $K$ is positive and relates to the crowding coefficient $q=(a-b) / K$ which acts to limit the birth rate when the density is high relative to the carrying capacity. Infection is directly transmitted with transmission coefficient $\beta$ and infected red squirrels experience additional mortality (virulence) at rate $\sigma$ due to the disease. There is no recovery class since SQPV is lethal to red squirrels (Tompkins et al., 2002).

Initially there are a small number of infected red squirrels so it is important to include the possibility of disease extinction or fade outs. Therefore we develop a stochastic version of the deterministic model in Equations (1) as this captures the random variability of infectious disease at low densities. Furthermore, in heterogeneous spatial environments the spread of infection may be dictated by rare dispersal events and spatial, probabilistic frameworks can highlight these key dispersal routes. Therefore, within each $1 \mathrm{~km}$ by $1 \mathrm{~km}$ landscape cell (Figure 1(b)) we represent the dynamics using a spatial, stochastic model. The rates of change in the deterministic model (1) are turned into probabilities of events (Table 1, see Renshaw (1993) and Keeling and Rohani (2008) for details on the methodology) which account for changes in abundance within each grid patch. In addition, the abundance within patches can change due to dispersal and disease spread between neighbouring cells.

We assume squirrels core movement has a range $\alpha \mathrm{km}$ (approximated as a circle with diameter of $0.3 \mathrm{~km}$ in this study (Bosch and Lurz, 2012)) which may include crossing cell boundaries. Therefore infection can occur through susceptible infected contact within a focal cell $(i, j)$ at rate $\beta S_{i, j} I_{i, j}$ and additionally through contact with neighbouring cells at a rate

$$
\beta S_{i, j}\left(\frac{\alpha}{2} \Sigma I_{a}+\frac{\alpha^{2}}{4} \Sigma I_{c}\right)
$$

where the $\Sigma I_{a}$ represents the contributions from the neighbouring adjacent cells and the $\Sigma I_{c}$ the corner cells, see section S3 of the Supplementary Information for more details. Core range activity is significantly less than the 


\begin{tabular}{lll}
\hline Description & $\operatorname{Outcome}$ & Probability \\
\hline Birth & $\operatorname{Pr}\left(S_{i, j} \rightarrow S_{i, j}+1\right)$ & $:\left[\left(a-q_{i, j} H_{i, j}\right) H_{i, j}\right] / R$ \\
Natural death of S & $\operatorname{Pr}\left(S_{i, j} \rightarrow S_{i, j}-1\right)$ & $:\left[b S_{i, j}\right] / R$ \\
Natural death of I & $\operatorname{Pr}\left(I_{i, j} \rightarrow I_{i, j}-1\right)$ & $:\left[b I_{i, j}\right] / R$ \\
Disease induced mortality & $\operatorname{Pr}\left(I_{i, j} \rightarrow I_{i, j}-1\right)$ & $:\left[\sigma I_{i, j}\right] / R$ \\
Dispersal of S & $\operatorname{Pr}\left(S_{i, j} \rightarrow S_{i, j}-1, S_{i^{*}, j^{*}} \rightarrow S_{i^{*}, j^{*}}+1\right)$ & $:\left[D_{i, j} S_{i, j}\right] / R$ \\
Dispersal of I & $\operatorname{Pr}\left(I_{i, j} \rightarrow I_{i, j}-1, I_{i^{*}, j^{*}} \rightarrow I_{i^{*}, j^{*}}+1\right)$ & $:\left[D_{i, j} I_{i, j}\right] / R$ \\
Transmission of disease & $\operatorname{Pr}\left(S_{i, j} \rightarrow S_{i, j}-1, I_{i, j} \rightarrow I_{i, j}+1\right)$ & $:\left[\beta S_{i, j}\left(\frac{\alpha^{2}}{4} \Sigma I_{c}+\frac{\alpha}{2} \Sigma I_{a}+I_{i, j}\right)\right] / R$ \\
\hline
\end{tabular}

Table 1: A list of all possible events in the model described in Section 2.2. The outcome of each event in cell $(i, j)$ is given in column 2 with the associated probability in column 3, where the rate (within square brackets) is divided by the total sum of the rates $R=\Sigma$ [rates] across all the cells. The dispersal of susceptible and infected individuals from the focal cell $(i, j)$ to a neighbouring cell $\left(i^{*}, j^{*}\right)$ is given by $D_{i, j}$ which is defined in Equation (3). The transmission of disease occurs when a susceptible individual comes into contact with an infected individual from the current cell $(i, j)$ or from 8 neighbouring cells where $\Sigma I_{c}$ and $\Sigma I_{a}$ are the total infected individuals in the corners and adjacent cells respectively.

grid-cell dimensions and individuals are likely to return to their focal cell and so we ignore these daily dispersal events. However we include long distance dispersals where an individual leaves the focal cell $(i, j)$ and enters a neighbouring cell $\left(i^{*}, j^{*}\right)$. We assume saturation dispersal such that individuals are more likely to disperse as cell density increase (which is appropriate for dispersal in mammals (Poethke and Hovestadt, 2002)) by the following function

$$
D_{i, j}=d \exp \left(\frac{-\left(K_{i, j}-\left(S_{i, j}+I_{i, j}\right)\right)}{0.5 K_{i, j}}\right)
$$

where $d$ is the dispersal coefficient when the cell population is at its carrying capacity. There is no available data on the frequency of long distance dispersals, therefore we make an assumption that a squirrel will make approximately one long range dispersal in its lifetime (when the density is at carrying capacity), thus giving $d=b$ (note that we do carry out sensitivity to this parameter in Section 6 in the Supplementary Information). Once an individual disperses from the focal cell it enters one of the eight neighbouring cells at random dependent on their cell density (with appropriate scaling between adjacent and corner cells).

Using methods of Gillespie (1977) (see also Keeling and Rohani (2008) and Renshaw (1993)) we transform the rates into probabilities by dividing each rate by the sum of the total rates $R=\Sigma$ [rates] (see Table 1). The choice of event is determined at random and the time between events is defined as $\delta t=-(\ln (\psi)) / R$ where $\psi$ is drawn from a uniform distribution between 0 and 1 (which assumes that time to the next event is an exponentially distributed random variable (Renshaw, 1993)). After each event grid cell densities and therefore probabilities are updated. By repeating this process the abundance of susceptible and infected individuals in each cell can be tracked over space and time. A more detailed description of the model process in given in Section S1 of the Supplementary Information. 


\subsection{Arran connectivity}

GIS data provides habitat information that is transformed into grid-cell carrying capacity and informs within cell epidemiological dynamics and between cell transmission and dispersal. In addition to the GIS data, groundtruthing in collaboration with Forestry Commission Scotland, has identified further routes of poor connectivity highlighting, for instance, exposed mountain tops that would be a barrier to dispersal. This can be incorporated in the model by preventing dispersal between appropriate cells (shown by black lines in Figure 1(b)). The ground-truthing information is likely to impact dispersal and disease spread predicted by our model since, for example, in the middle of the island a barrier in Figure 1(b) prevents east to west connectivity. In this study we compare two connectivity scenarios: the first includes all the connectivity information obtained from GIS and ground-truthing, whilst the second uses GIS data only. It is likely that the connectivity in most spatial models utilise GIS information only, so in comparing the two scenarios we aim to highlight the importance of incorporating local knowledge (if available) when modelling disease spread.

\subsection{Parameter Values}

All demographic parameters are kept constant. The natural death rate, $b=0.9 \mathrm{yr}^{-1}$, is produced using adult mortality estimates presented in (Barkalow et al., 1970). The growth rate for red squirrels is estimated at $0.6 \mathrm{yr}^{-1}$ (Tompkins et al., 2003) which translates into a birth rate $a=1.5 \mathrm{yr}^{-1}$. The values of $a$ and $b$ combine with grid-cell dependant carrying capacities to determine the susceptibility to crowding $q$ in each patch. As detailed in Section 2.2 dispersal rate $d=b$ and core movement has a range $\alpha=0.3 \mathrm{~km}$ (Bosch and Lurz, 2012).

Table 2 displays the four sets of parameter values used for evaluating disease spread on Arran (note that the non-disease related parameters remain fixed). In the absence of detailed information on red-red squirrel transmission rates of SQPV, previous modelling studies have simulated a range of potential values (e.g. Rushton et al. $(2000,2006)$ where encounter rates were density dependent and values for the likelihood of infection for individuals were chosen by sampling deviates from a uniform range of 0-1); or have chosen grey-grey transmission values derived from anti-body blood sampling data (Sainsbury et al., 2000) as a benchmark value (e.g. Tompkins et al. (2003); White et al. (2014)) and in each case undertook sensitivity analyses to assess the impacts of assumption. Here we follow the latter approach and use grey-grey squirrel transmission (acute, low transmission in Table 2) values as a lower benchmark and explore the implications of higher transmission scenarios. Due to the uncertainty of red-red transmission rates we also consider an acute disease with high transmission (Table $2)$.

The variability in the disease spread has been linked to disease characteristics (Cross et al., 2005; Hess, 1996). For example Cross et al. (2005) found that a slow, chronic infection generally resulted in an increased number of infecteds when compared to a fast, acute infection. To provide a general understanding of disease spread on our 


\begin{tabular}{|l|c|c|c|c|}
\hline \multirow{2}{*}{ Parameter } & \multicolumn{2}{|c|}{ Acute (high virulence) } & \multicolumn{2}{c|}{ Chronic (low virulence) } \\
\cline { 2 - 4 } & $\begin{array}{c}\text { Low } \\
\text { transmission }\end{array}$ & $\begin{array}{c}\text { High } \\
\text { transmission }\end{array}$ & $\begin{array}{c}\text { Low } \\
\text { transmission }\end{array}$ & $\begin{array}{c}\text { High } \\
\text { transmission }\end{array}$ \\
\hline Natural birth rate, $a$ & 1.5 & 1.5 & 1.5 & 1.5 \\
Natural death rate, $b$ & 0.9 & 0.9 & 0.9 & 0.9 \\
Core movement range, $\alpha$ (in $\mathrm{km})$ & 0.3 & 0.3 & 0.3 & 0.3 \\
Dispersal rate, $d$ & $0.9 *$ & $0.9 *$ & 0.9 & 0.9 \\
Transmission coefficient, $\beta\left(\mathrm{km}^{2} \mathrm{yr}^{-1}\right)$ & 0.6 & 12 & 0.6 & 12 \\
Disease induced death, $\sigma$ & 26 & 26 & 0.445 & 0.445 \\
\hline
\end{tabular}

Table 2: The disease parameters (in years ${ }^{-1}$ unless otherwise stated) considered in this study. The models sensitivity to dispersal rate $d$ has been tested in S6 of the Supplementary Information for the parameter sets denoted by $*$.

complex landscape we therefore consider disease parameters that represent chronic infections (low virulence) with low and high transmissibility (Table 2). Here virulence is chosen to maintain the same basic reproductive rate of the disease for the acute, high transmission and chronic, low transmission cases. Establishing these parameter sets allows us to examine the impact of different infectious disease characteristics on the spread and persistence of infection in fragmented habitats. (For further details on parameter estimation see Section S3 of the Supplementary Information.)

\section{Results}

\subsection{Squirrelpox spread on Arran}

Assuming that SQPV transmission between red squirrels is the same as SQPV tranmission between grey squirrels (acute, low transmission in Table 2) the spread of SQPV from its source of introduction is limited (Figure 2). This indicates that there may be no large scale threat to the population of red squirrels on Arran if an SQPV outbreak occurred. In most simulations the disease fails to spread and fades out within 2 months of the initial introduction (Figure 2(a)). In a few simulations (4 out of 25) there is limited disease spread to neighbouring cells but nevertheless the disease fades out within 6 months of the initial introduction (Figure 2(c)). In all cases the population reduction due to the disease is relatively small (infection within a grid-cell reduces the density by at most 25\%). Therefore, assuming the grey-grey parameter set for SQPV does not permit the spread of disease or cause any significant reduction to the red squirrel population on Arran. This is due to the high virulence and low transmissibility of the disease which in combination with the relatively low densities on Arran (around 0.1-0.5 squirrels ha ${ }^{-1}$ around Brodick) means the basic reproductive rate of the disease $\left(R_{0}\right)$ is not sufficiently high to promote large-scale disease spread or persistence (see Supplementary Information for a spatial analysis of $\left.R_{0}\right)$. Since disease spread is limited with these parameter values there is no difference between the scenarios using GIS and ground-truthing and GIS information only. 

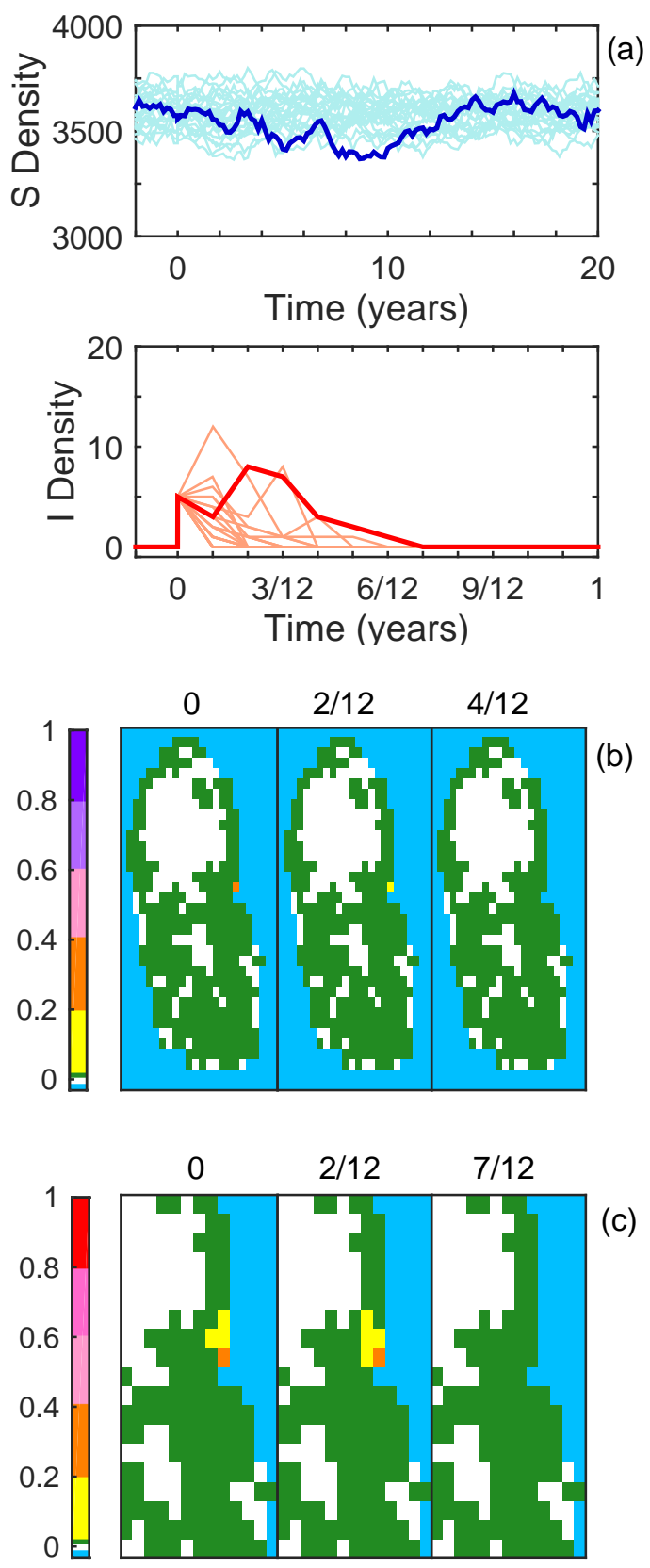

Figure 2: The model using grey-grey SQPV parameters (acute, low transmission in Table 2) where connectivity includes GIS information and ground-truthing. Once the total population has achieved quasi-steady state, 5 infected red squirrels are introduced at Brodick (Figure 1(a)) at time 0. In (a) the total susceptible (blue) and infected (red) population densities are shown against time for 25 model realisations with a single realisation highlighted. In (b) and (c), snap shots show the spatial distribution of disease at different time frames (identified, in years, in the title of each frame). We define the disease prevalence as the proportion of infected hosts in each cell $\left(\operatorname{Prev}=\left(I_{i, j}\right) /\left(I_{i, j}+S_{i, j}\right)\right)$. In (b) we show the proportion of simulations that have a prevalence greater than 0.2. In (c) we show the prevalence for the realisation highlighted in (a) with an enlarged scale to focus on the region where the disease was introduced.

\subsection{Acute infection with high transmissibility}

Figure 3 shows the population dynamics and disease spread when the high virulence of SQPV is retained and transmission is increased (acute, high transmission parameters in Table 2). When restrictions to dispersal 
GIS and Ground Truthing
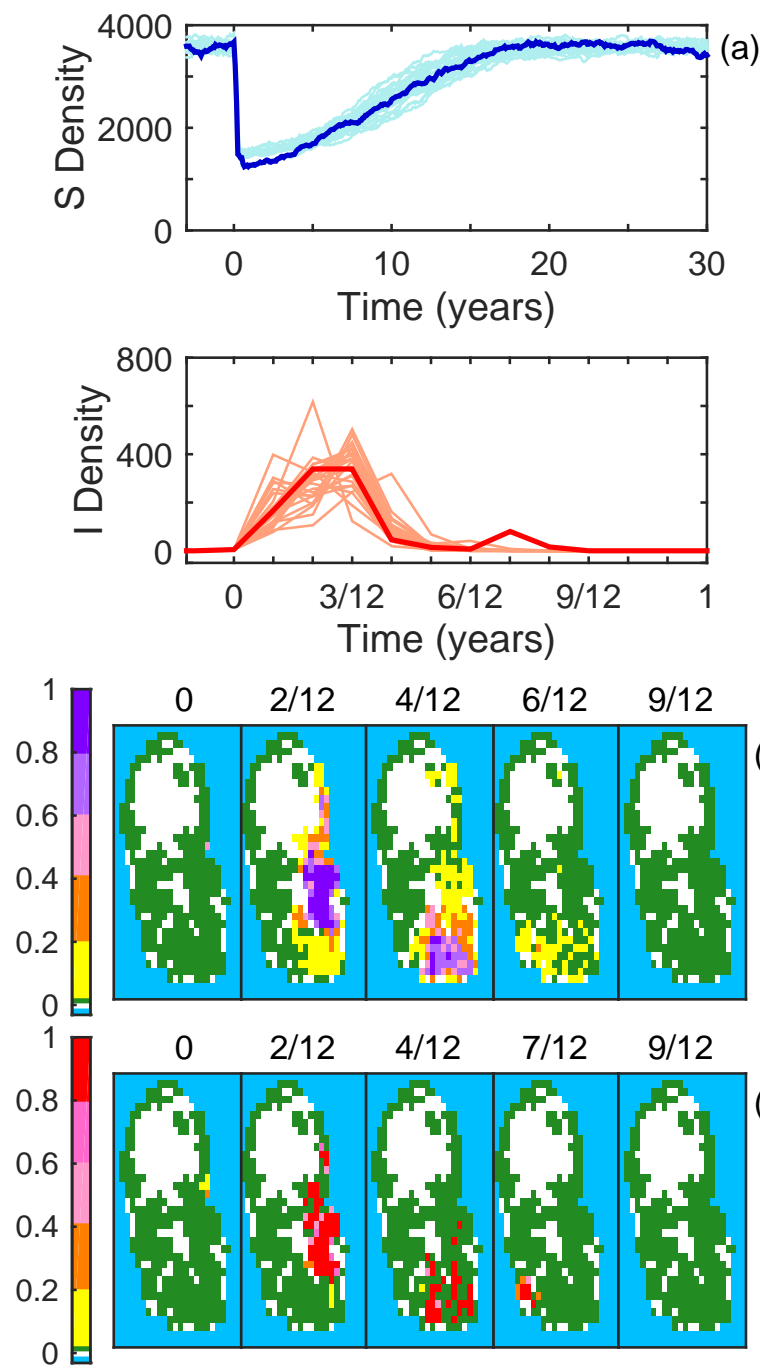

GIS
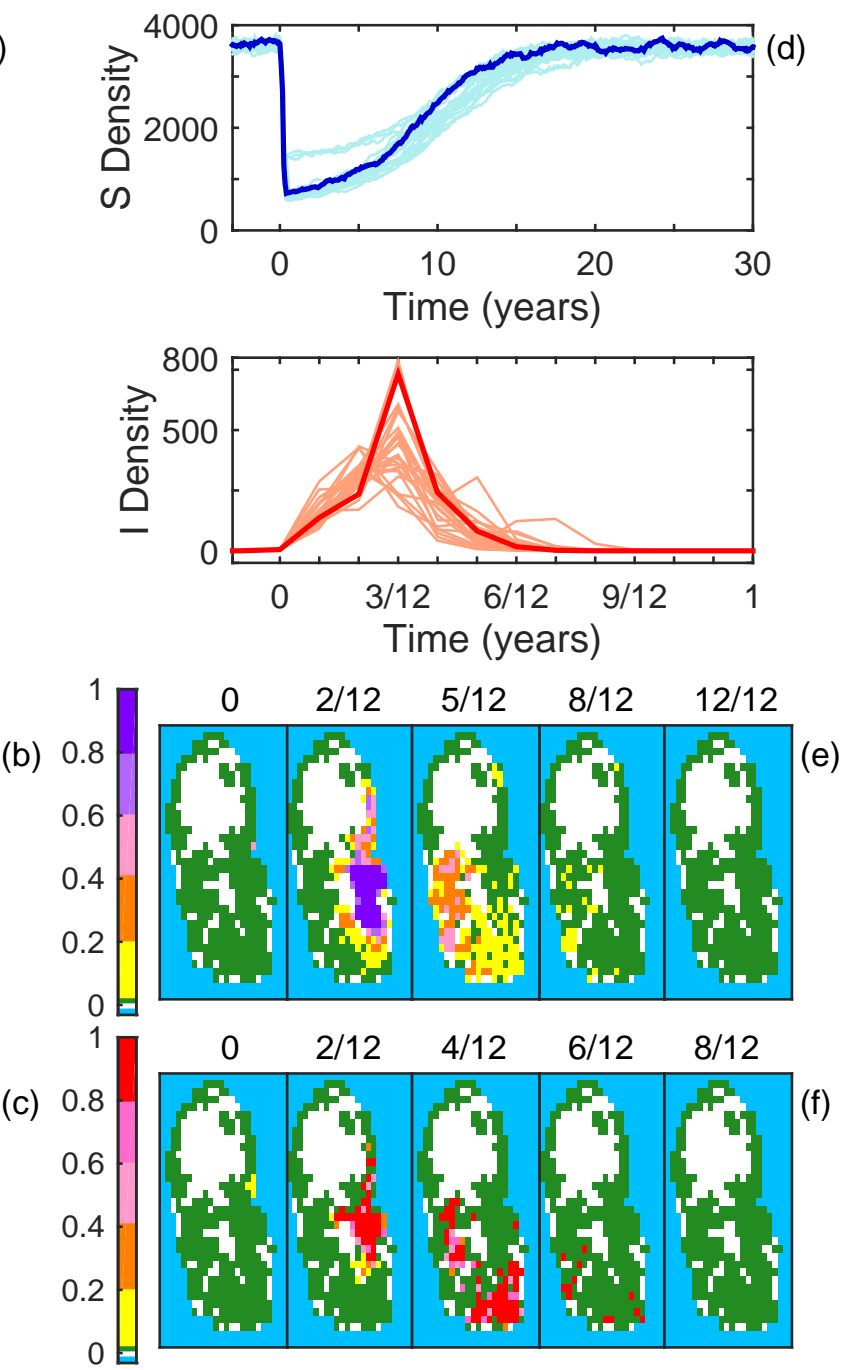

Figure 3: The model with acute, high transmission parameters (Table 2) where in (a)-(c) connectivity includes GIS information and ground-truthing and in (d)-(f) GIS information only. 5 infected red squirrels are introduced at Brodick (Figure 1(a)) at time 0. In (a) and (d) the total susceptible (blue) and infected (red) population densities are shown against time for 25 model realisations with a single realisation highlighted. In (b), (c), (e) and (f), snap shots show the spatial distribution of disease at different time frames (identified, in years, in the title of each frame). (b) and (e) show the proportion of simulations that have a prevalence greater than 0.2. (c) and (f) show the prevalence for the realisation highlighted in (a)and (c) respectively. Further description is given in Figure 2 caption.

imposed by ground-truthing are included the disease spreads rapidly throughout the east and south of the island (region 1 in Figure 1(b)). Disease outbreaks lead to a substantial reduction of up to $89 \%$ of local density. The density in region 1 is reduced to $15 \%$ and the total population is reduced to $42 \%$ of its pre-infection levels. The wavelike expansion in the distribution of the disease and the eventual containment of disease is dependent on areas of high and low landscape connectivity. In areas of high connectivity the disease spreads quickly causing high mortality, however upon reaching regions of low connectivity the disease may fade out as it rapidly exhausts the local pool of susceptible individuals and is rarely able to spread over poor quality (low density) habitat (this 
prevents the spread from region 1 to 2 as denoted in Figure 1(b)). When the additional barriers to dispersal imposed by ground-truthing are removed the disease spread is more extensive and occurs across the majority of the island (since the connectivity between regions 1 and 2 is improved). There is high disease mortality in regions 1 and 2 and the total population is reduced to $22 \%$ of its pre-infection levels. Poor connectivity between regions 2 and 3 prevent disease outbreaks in region 3 . In both connectivity scenarios the disease fades out following an epidemic. Locally this takes 1-2 months and typically fades out on the entire island within 7 months. Disease fade out arises from the rapid spread of the highly virulent disease, which, largely exhausts the local population of susceptibles. Following the disease fade out the population recovers to its pre-infection level through demographic processes. These simulations indicate that a highly virulent, highly transmissible disease can have marked impacts on the population density over a large scale. They also reveal how areas of low connectivity, through which the disease fails to spread, can protect populations from disease.

\subsection{Chronic infection with low transmissibility}

Figure 4 shows the impact of a chronic infection with low transmission (Table 2) when connectivity includes the information from ground-truthing (note, the basic reproductive rate of the disease, $R_{0}$, is the same in Figures 3 and 4, see Supplementary Information for further details). The disease initially spreads throughout region 1 reducing the population here to $17 \%$ of pre-infection levels, with the total population reduced to $43 \%$. This outcome is similar to the acute, high transmission scenario with connectivity from GIS and ground-truthing (Figure 3(a-c)) except for the chronic disease the rate of spread is reduced. The key difference is that the disease persists under this chronic scenario in the long-term therefore a rare dispersal event eventually allows the disease to expand into region 2 in $80 \%$ and region 3 in $67 \%$ of realisations. Although, the disease persists in the long-term in a single realisation of the model the distribution of infection changes over time with periodic epidemics disease and fade out locally (Figure $4(\mathrm{c})$ ). (Results observed in the connectivity scenario in which the dispersal barriers imposed through ground-truthing are removed show a similar reduction to the host densities, but here the disease initially spreads to all regions.) A key difference between Figure 3 and Figure 4 is that the persistence of the chronic infection provides greater opportunity for dispersal and therefore it can eventually expand over a more extensive region.

For the remaining scenario, a chronic infection and high transmission in Table 2, the reproductive rate of the disease is greatly increased. Here, the disease spreads rapidly across the whole landscape, the total population abundance is reduced dramatically and most individuals become infected. This leads to the local extinction of populations. In the long term there are two possible outcomes. Either the entire population becomes extinct or the disease becomes extinct and the population recovers to pre-infection levels (for more information see Section S5.3 in the Supplementary Information).

Results for all the scenarios presented here but with the low red squirrel density estimates used to determine 
grid-cell carrying capacities, are reported in Section S5 of the Supplementary Information. The results for the low density scenarios are qualitatively similar to those for the high density scenarios although the effect of the lower density does reduce the severity of disease outbreaks and extent of disease spread (as at lower density the reproductive ratio of the disease, $R_{0}$, is reduced).

We noted in Section 2.2 that there is a lack of available data for determining the frequency of long distance dispersals thus we set $d=b$ (Table 2) which assumes that on average a squirrel is likely to make one long range dispersal in its lifetime. In section S6 of the Supplementary Information we carrying out sensitivity analysis of parameter $d$ for acute, low transmission and acute, high transmission parameter sets. We show that for both an increase and decrease of the dispersal coefficient, the disease pattern in qualitatively similar to the results shown for the baseline parameter set. The lack of sensitivity to the dispersal parameter suggests that disease transmission may be driven by local spread within populations rather than the long distance movement of infected individuals.

\section{Discussion}

Emerging infectious diseases (EIDs) and disease-mediated invasions are a substantial threat to native wildlife abundance and diversity (Daszak, 2000; Daszak et al., 2001; Morse, 1995). Through the use of mathematical models we addressed how infectious disease characteristics and landscape connectivity combined to influence disease spread and persistence. The model analysis allowed us to make system specific predictions on the potential threat of SQPV to red squirrels on the Isle of Arran that are being used to influence conservation management decisions (Macpherson et al., 2013). Further, the generality of our model framework allowed us to uncover broadly applicable findings on how different infection characteristics (acute or chronic infections) influence the spread and persistence of disease in realistic landscapes.

To protect the remaining populations of red squirrels 35 red squirrel strongholds in England and Scotland have been developed in which reds are isolated from grey squirrels (through control of greys or due to the isolated nature of the stronghold). However, SQPV remains a threat to stronghold red populations. For instance, high density red populations in Formby, Merseyside and in Whinfell, Cumbria have been protected by trapping and removal of greys since the launch of strongholds in 2006 (Parrott et al., 2009; Chantrey et al., 2014), but have suffered repeated SQPV outbreaks which has resulted in a marked reduction in population abundance (followed by the disease fading out and subsequent population increases). We argue that the simulated results using the landscape of Arran, despite being an island, are also representative of the mainland strongholds. A buffer area of trapping around the mainland strongholds removes greys, thus it is unlikely that there will be more than one challenge at a time (although several may occur over a period of time) making our simulations a reasonable representation. Using grey-grey SQPV parameters, the model predicts the impact of disease on 
the low density populations on Arran will be limited to a short term, local outbreak focused at the point of disease introduction. When considering a highly virulent, highly transmissible disease (which could represent SQPV if red-red transmission is sufficiently higher than the grey-grey level or could represent a system where grey-grey transmission is accurate for SQPV in red squirrels and the population density is higher) the disease spread is more widespread and a substantial reduction in population density is predicted due to disease induced mortality. However, disease spread does not occur between poorly connected regions and the disease fades out leading to population recovery to pre-infection levels - supporting the observations of disease dynamics in high density stronghold populations (Parrott et al., 2009). In terms of the conservation of red squirrels on Arran the model suggests that if introduced, SQPV spread (using grey-grey transmission parameters) is likely to remain local to the point of introduction and will not cause an island-wide epidemic and as long as grey squirrels are removed from the island reds should recover.

The model results have broad implications for red squirrel conservation and more generally in the understanding of the spread of highly virulent EIDs through native populations. Our results support previous studies Duff et al. (2010) and Gurnell et al. (2006) who concluded that in the absence of grey squirrels SQPV would not persist due to the increased mortality of reds reducing the chance of disease transmission. This suggests that the disease cannot persist without an additional local reservoir of infection and emphasises that limiting grey squirrel introductions and/or preventing the further population expansion of grey squirrels may be critical to safe-guarding the remaining red squirrel populations in the UK. The interface between the distribution of red and grey squirrels in the UK occurs in Highland Scotland and has been relatively stable for 40 years. Grey populations to the south of this interface are currently disease-free but recent surveys (Scottish Wildlife Trust, 2013) indicate that SQPV is spreading northwards from Southern Scotland and so there is a clear risk that SQPV will spread to grey populations at the interface. The model results here suggest the SQPV may cause epidemic outbreaks and mortality in red populations adjacent to the interface but that it is unlikely to spread extensively through the red (only) populations in Highland Scotland (north of the interface). However, the impact of the disease will increase the apparent competition from greys at the interface (Tompkins et al., 2003) and could allow greys to expand their distribution northwards (Bell et al., 2008). This would have detrimental implications for the conservation of red squirrels and it is therefore essential that it is a focus for further study.

The implication of landscape connectivity for wildlife viability and persistence is well studied with clear evidence that increased connectivity can increase population abundance and prevent extinction (Dobson et al., 1999; Fahrig and Merriam, 1985; Henein and Merriam, 1990; Lookingbill et al., 2010; Schumaker, 1996; van Andel and Aronson, 2012). However, it has been argued that increased connectivity may also promote the spread of infectious disease (Bienen, 2002; Hess, 1994; Real and Biek, 2007). Previous theoretical studies have used idealised spatial set-ups to examine the impact of introduced infectious disease in fragmented landscapes. These studies have synthesized techniques from mathematical epidemiology with metapopulation models (Hess, 
1996; McCallum and Dobson, 2002) or developed stochastic simulation models based on underlying epidemiological frameworks that represent the dynamics on an array of patches connected in a simplified manner (Hess, 1996; White et al., 2014). The most similar study to ours used an individual-based, stochastic disease model to represent the dynamics on a two-dimensional lattice of identical patches with nearest neighbour dispersal (Cross et al., 2005). Findings indicate that improved connectivity can enhance the spread and persistence of disease, although the benefits of improved connectivity for population persistence may outweigh the disease risks (McCallum and Dobson, 2002). Also, local population interactions combine with infectious disease characteristic such that acute, highly virulent, infection may fade-out in a population whereas chronic, low virulence, infection may be supported (Hess, 1996; Cross et al., 2005).

Our study - which uses a realistic, heterogeneous, landscape based on habitat data - supports these findings. When the basic reproductive rate of the disease, $R_{0}$, is low (due to a combination of a low density habitat, high virulence and low transmission) the disease fails to persist and spread is limited. When $R_{0}$ is increased disease spread is more marked and the long-term impact depends on the nature - acute or chronic - of the disease. In particular, acute, highly transmissible infections can spread rapidly through regions of good habitat, but may fail to spread between poorly connected regions. The associated high virulence leads to severe population crashes and the disease therefore burns-out allowing populations to recover to pre-infection levels. Chronic infections spread more slowly, but can persist in regions of good habitat. This disease persistence provides increased opportunities for the disease to spread between poorly connected regions and therefore chronic infections are more widespread. By using a realistic landscape we have, therefore, been able to understand how high and low connectivity and fragmentation interact.

Evidence for different acute and chronic infection outbreaks have been identified in real-life systems. For example, epidemic outbreaks followed by the disease fading out has been observed in the high density red squirrel population in the stronghold at Formby, Merseyside (Parrott et al., 2009; Chantrey et al., 2014). Alternatively, the squirrel Adenovirus may have similarities with the results of the chronic infection since it is less virulent than SQPV but can cause mortality when associated with other stress factors. Adenovirus has been reported in natural red squirrel populations that are free from grey squirrels with the disease persisting for many years (Everest et al., 2013; Martínez-Jiménez et al., 2011). Detailed investigations into the prevalence of Adenovirus in red populations is ongoing (Everest et al., 2013) but our modelling results suggest that the chronic nature of the disease may allow it to spread and persist should it be introduced into a naive population - such as the red population on Arran.

The results of our study highlight how wildlife management strategies should carefully consider the risk of disease outbreaks. Management strategies that increase population connectivity or increase population density (e.g. through supplementary feeding or changes to forest composition) may increase the risk of disease spread and so scale and location of such measures should be considered carefully. Our study has shown how stochastic 
modelling frameworks incorporating realistic habitat data are essential tools for the evaluation of such management strategies, and have wide application in the development of conservation planning, for both population stability and disease resilience. 


\section{References}

Barkalow, F., Hamilton, R., and Soots Jr, R. (1970). The vital statistics of an unexploited gray squirrel population. The Journal of Wildlife Management, pages 489-500.

Beier, P. and Noss, R. (1998). Do habitat corridors provide connectivity? Conservation Biology, 12(6):12411252.

Bell, S. S., White, A., Sherratt, J. a., and Boots, M. (2008). Invading with biological weapons: the role of shared disease in ecological invasion. Theoretical Ecology, 2(1):53-66.

Bienen, L. (2002). Informed decisions conservation corridors and the spread of infectious disease. Conservation in practice, $3(2): 10-17$.

Bosch, S. and Lurz, P. W. W. (2012). The Eurasian Red Squirrel. Westarp Wissenschaften.

Chantrey, J., Dale, T., Read, J., White, S., Whitfield, F., Jones, D., McInnes, C., and Begon, M. (2014). European red squirrels population dynamics driven by squirrelpox at a gray squirrel invasion interface. Ecology and Evolution, 4:3788-3799.

Chesser, R. and Ryman, N. (1986). Inbreeding as a strategy in subdivided populations. Evolution, 40(3):616624.

Cross, P. C., Lloyd-Smith, J. O., Johnson, P. L., and Getz, W. M. (2005). Duelling timescales of host movement and disease recovery determine invasion of disease in structured populations. Ecology Letters, 8(6):587-595.

Daszak, P. (2000). Emerging Infectious Diseases of Wildlife- Threats to Biodiversity and Human Health. Science, 287(5452):443-449.

Daszak, P., Cunningham, a. a., and Hyatt, a. D. (2001). Anthropogenic environmental change and the emergence of infectious diseases in wildlife. Acta tropica, 78(2):103-16.

Dobson, A., Ralls, K., Foster, M., Soule, M. E., Simberloff, D., Doak, D., Estes, J. A., Mills, L. S., Mattson, D., Dirzo, R., et al. (1999). Corridors: reconnecting fragmented landscapes. Continental conservation: Scientific foundations of regional reserve networks, pages 129-170.

Duff, J., Holmes, J., and Barlow, A. (2010). Surveillance turns to wildlife. Veterinary Record, 167:154-156.

Everest, D. J., Butler, H., Blackett, T., Simpson, V. R., and Shuttleworth, C. M. (2013). Adenovirus infection in red squirrels in areas free from grey squirrels. The Veterinary record, 173(8):199-200.

Fahrig, L. and Merriam, G. (1985). Habitat Patch Connectivity and Population Survival. Ecology, 66(6):17621768. 
Gillespie, D. (1977). Exact stochastic simulation of coupled chemical reactions. The journal of physical chemistry, 93555(1):2340-2361.

Gurnell, J., Rushton, S., Lurz, P., a.W. Sainsbury, Nettleton, P., Shirley, M., Bruemmer, C., and Geddes, N. (2006). Squirrel poxvirus: Landscape scale strategies for managing disease threat. Biological Conservation, 131(2):287-295.

Hanski, I. (1998). Metapopulation dynamics. Nature, 396(6706):41-49.

Hanski, I. and Gilpin, M. (1991). Metapopulation dynamics: brief history and conceptual domain. Biological Journal of the Linnean Society, 42:3-16.

Henein, K. and Merriam, G. (1990). The elements of connectivity where corridor quality is variable. Landscape Ecology, 4(2-3):157-170.

Hess, G. (1994). Conservation corridors and contagious disease: a cautionary note. Conservation Biology, $8(1): 256-262$.

Hess, G. (1996). Disease in metapopulation models: implications for conservation. Ecology, pages 1617-1632.

Joint Nature Conservation Committee (2012). Uk biodiversity action plan.

Kareiva, P. and Wennergren, U. (1995). Connecting landscape patterns to ecosystem and population processes. Nature, 373:299-302.

Keeling, M. J. (1999). The effects of local spatial structure on epidemiological invasions. Proceedings of the Royal Society of London. Series B: Biological Sciences, 266(1421):859-67.

Keeling, M. J. and Rohani, P. (2008). Modeling infectious diseases in humans and animals. Princeton University Press.

Levins, R. (1969). Some demographic and genetic consequences of environmental heterogeneity for biological control. Bulletin of the ESA, 15(3):237-240.

Lookingbill, T. R., Gardner, R. H., Ferrari, J. R., and Keller, C. E. (2010). Combining a dispersal model with network theory to assess habitat connectivity. Ecological applications: a publication of the Ecological Society of America, 20(2):427-41.

Lurz, P., Geddes, N., and Lloyd, A. (2003). Planning a red squirrel conservation area: using a spatially explicit population dynamics model to predict the impact of felling and forest design plans. Forestry, 76(1):93-106.

Lurz, P. W. W. (1995). Ecology and conservation of the red squirrel (Sciurus vulgaris L.) in upland conifer plantations. $\mathrm{PhD}$ thesis, University of Newcastle. 
Lurz, P. W. W. (2012). Arran Red Squirrel Assessment, Unpublished. Technical report.

Macpherson, M., Davidson, R., Duncan, D., White, A., Lurz, P., and Jarrett, A. (2013). Assessing the impact of the squirelpox virus on Eurasian Red squirrel, Unpublished. Technical report.

Manchester, S. and Bullock, J. (2000). The impacts of non-native species on UK biodiversity and the effectiveness of control. Journal of Applied Ecology, 37:845-864.

Martínez-Jiménez, D., Graham, D., Couper, D., Benkö, M., Schöniger, S., Gurnell, J., and Sainsbury, A. W. (2011). Epizootiology and pathologic findings associated with a newly described adenovirus in the red squirrel, Sciurus vulgaris. Journal of wildlife diseases, 47(2):442-54.

McCallum, H. and Dobson, A. (2002). Disease, habitat fragmentation and conservation. Proceedings of the Royal Society of London. Series B: Biological Sciences, 269(1504):2041-2049.

Morse, S. S. (1995). Factors in the emergence of infectious diseases. Emerging infectious diseases, 1(1):7-15.

Ostfeld, R. S., Glass, G. E., and Keesing, F. (2005). Spatial epidemiology: an emerging (or re-emerging) discipline. Trends in ecology $\&$ evolution, 20(6):328-36.

Parrott, D., Quy, R., Driel, K. V., and Lurz, P. (2009). Review of red squirrel conservation activity in northern England. Technical Report September, Natural England.

Patz, J. a., Daszak, P., Tabor, G. M., Aguirre, a. A., Pearl, M., Epstein, J., Wolfe, N. D., Kilpatrick, a. M., Foufopoulos, J., Molyneux, D., Bradley, D. J., and Emergence, D. (2004). Unhealthy Landscapes: Policy Recommendations on Land Use Change and Infectious Disease Emergence. Environmental Health Perspectives, 112(10):1092-1098.

Poethke, H. J. and Hovestadt, T. (2002). Evolution of density- and patch-size-dependent dispersal rates. Proceedings of the Royal Society of London. Series B: Biological Sciences, 269(1491):637-45.

Real, L. a. and Biek, R. (2007). Spatial dynamics and genetics of infectious diseases on heterogeneous landscapes. Journal of the Royal Society Interface, 4(16):935-48.

Renshaw, E. (1993). Modelling biological populations in space and time, volume 11. Cambridge University Press.

Riley, S. (2007). Large-scale spatial-transmission models of infectious disease. Science (New York, N.Y.), 316(5829):1298-301.

Rushton, S., Lurz, P., Gurnell, J., and Fuller, R. (2000). Modelling the spatial dynamics of parapoxvirus disease in red and grey squirrels: a possible cause of the decline in the red squirrel in the UK? Journal of Applied Ecology, 37(6):997-1012. 
Rushton, S., Lurz, P., Gurnell, J., Nettleton, P., Bruemmer, C., Shirley, M., and Sainsbury, A. (2006). Disease threats posed by alien species: the role of a poxvirus in the decline of the native red squirrel in britain. Epidemiology and Infection, 134(03):521-533.

Sainsbury, A. W., Nettleton, P., Gilray, J., and Gurnell, J. (2000). Grey squirrels have high seroprevalence to a parapoxvirus associated with deaths in red squirrels. Animal Conservation, 3(03):229-233.

Schumaker, N. (1996). Using landscape indices to predict habitat connectivity. Ecology, 77(4):1210-1225.

Scottish Wildlife Trust (2013). Annual Report and Financial Statements for the year ended 31st March 2013. Technical Report March.

Smith, K. F., Sax, D. F., and Lafferty, K. D. (2006). Evidence for the role of infectious disease in species extinction and endangerment. Conservation biology, 20(5):1349-57.

Tompkins, D. M., Sainsbury, A. W., Nettleton, P., Buxton, D., and Gurnell, J. (2002). Parapoxvirus causes a deleterious disease in red squirrels associated with uk population declines. Proceedings of the Royal Society of London. Series B: Biological Sciences, 269(1490):529-533.

Tompkins, D. M., White, A. R., and Boots, M. (2003). Ecological replacement of native red squirrels by invasive greys driven by disease. Ecology Letters, 6(3):189-196.

Tonkin, J. M. (1983). Ecology of the red squirrel (Sciurus vulgaris) in mixed woodland. PhD thesis, University of Bradford.

Travis, J. M. J. (2003). Climate change and habitat destruction: a deadly anthropogenic cocktail. Proceedings of the Royal Society of London. Series B: Biological Sciences, 270(1514):467-73.

van Andel, J. and Aronson, J. (2012). Restoration ecology: the new frontier. John Wiley \& Sons.

Westervelt, J. D., Shapiro, M., Goran, W. D., and Gerdes, D. P. (1992). Geographic Resources Analysis Support System (GRASS) Version 4.0 User's Reference Manual. US Army Corps of Engineers, Construction Engineering Research laboratory.

White, A., Bell, S. S., Lurz, P. W., and Boots, M. (2014). Conservation management within strongholds in the face of disease-mediated invasions: red and grey squirrels as a case study. Journal of Applied Ecology.

Worrell, R. (2012). An Assessment Of The Potential Impacts Of Ash Dieback In Scotland. Technical Report January, The Forestry Commission, Edinburgh. 

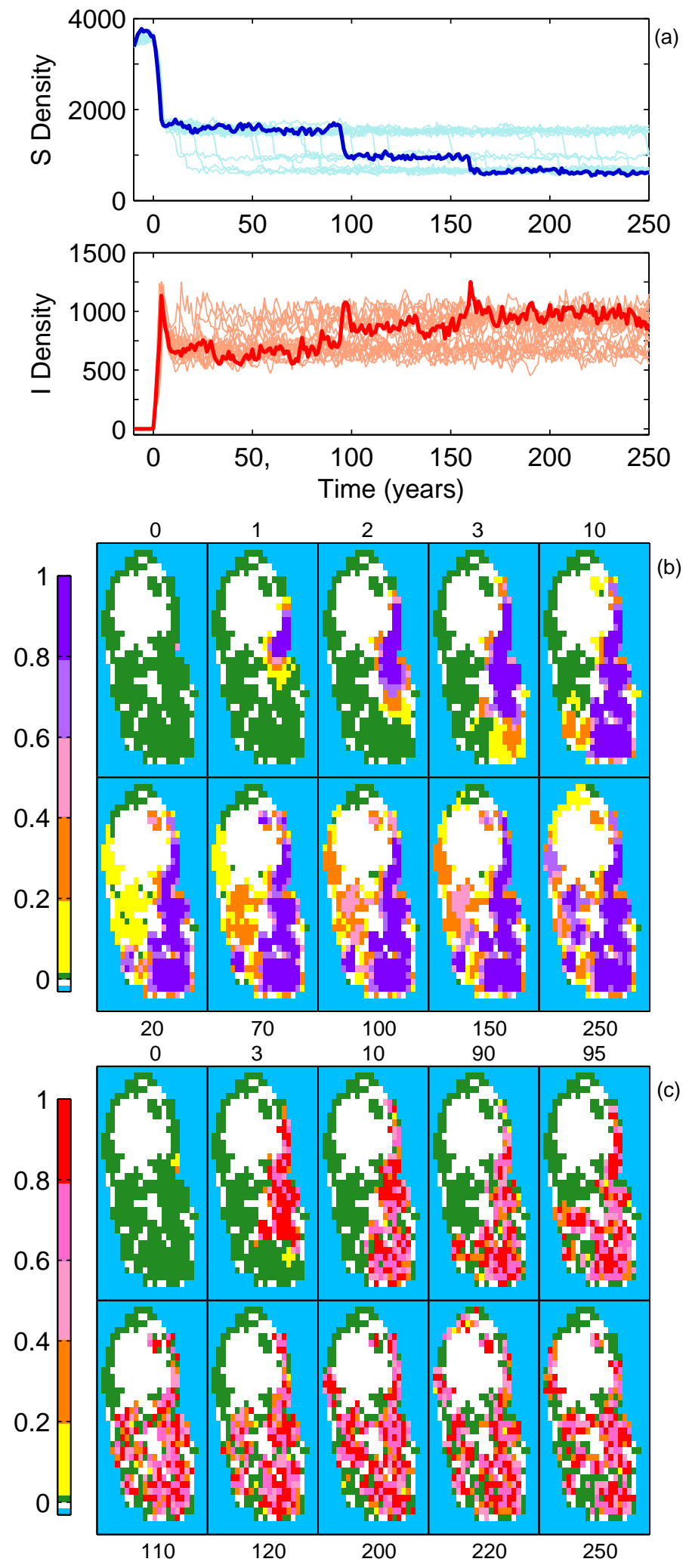

Figure 4: The model with chronic, low transmission parameters (Table 2) where connectivity includes GIS information and ground-truthing. 5 infected red squirrels are introduced at Brodick (Figure $1(\mathrm{a})$ ) at time 0. In (a) the total susceptible (blue) and infected (red) population densities are shown against time for 25 model realisations with a single realisation highlighted. In (b) and (c) snap shots show the spatial distribution of disease at different time frames (identified, in years, in the title of each frame) with (b) showing the proportion of simulations that have a prevalence greater than 0.2 and (c) showing the prevalence for the realisation highlighted in (a). Further description is given in Figure 2 caption. 